\title{
RNA-Seq Analysis of Activated PBMC Treated With LMWF5A Predicts an Anti-Inflammatory Mode of Action and Similar Drug Targets to Dexamethasone
}

Elizabeth D. Frederick ( $\boldsymbol{\nabla}$ efrederick@ampiopharma.com )

Ampio Pharmaceuticals https://orcid.org/0000-0001-5596-5094

Melissa A. Hausburg

Swedish Medical Center

Gregory W. Thomas

Ampio Pharmaceuticals

David Bar-Or

Swedish Medical Center

\section{Research Article}

Keywords: Dexamethasone, steroid, anti-inflammatory, osteoarthritis, COVID-19, PBMC

Posted Date: December 21st, 2021

DOI: https://doi.org/10.21203/rs.3.rs-790987/v2

License: (c) (i) This work is licensed under a Creative Commons Attribution 4.0 International License.

Read Full License 


\section{Abstract}

Background: The low molecular weight fraction of human serum albumin (LMWF5A) has immunomodulatory activity via its effects on multiple inflammatory mediators and is currently being evaluated for the treatment of hyperactive or persistent inflammatory conditions. To gain further insight into the mechanism of action (MOA) of LMWF5A, an investigation of its effects on activated immune cells was performed.

Methods and Results: Peripheral blood mononuclear cells (PBMC) were treated with vehicle control or LMWF5A and stimulated with lipopolysaccharide (LPS), LPS/interferon $\gamma$, or interleukin (IL)-4/IL-13, and RNAseq was performed to determine differentially expressed genes (DEGs) within each condition. Unbiased Ingenuity Pathway Analysis (IPA) of DEGs revealed anti-inflammatory and pro-resolving activities for LMWF5A. Moreover, comparison to all IPA upstream regulators predicted that the LMWF5A MOA is opposite to pro-inflammatory regulators and significantly matches the activity of several antiinflammatory molecules. These analyses identified the glucocorticoid dexamethasone (DEX) as the most significantly similar regulator to LMWF5A. To further explore similarities to DEX, LMWF5A DEGs were compared to two publicly available datasets of activated, DEX-treated PBMC. These comparisons showed continuity between predicted upstream regulators, affording further support to the hypothesis that LMWF5A acts in a manner like DEX. Nevertheless, not all LMWF5A-targeted DEGs showed directional regulation identical to DEX.

Conclusions: This study further defines the MOA of LMWF5A and provides hypotheses for future investigations. Because of its predicted similar biological effects and known safety profile, LMWF5A could potentially be used to treat conditions that are supported for DEX with fewer or less harmful side effects.

\section{Introduction}

Acute and chronic inflammation-associated diseases are characterized by dysregulated, excessive, and/or sustained immune activation. In general, aberrant activation entails increased expression of proinflammatory proteins, which may arise from enhanced pro-inflammatory transcription factor activity [1, 2]. Previous research has shown that the low molecular weight fraction of human serum albumin $\left(\right.$ LMWF5A, Ampion ${ }^{\mathrm{TM}}$ ) modulates levels of cytokines and other inflammatory mediators in various cell models [3]. Much research on the biologic drug LMWF5A has focused on its ability to inhibit release of the pro-inflammatory cytokine tumor necrosis factor (TNF) from immune cells, but LMWF5A has also been shown to affect interleukin (IL)-1 $\beta$, IL-6, IL-10, IL-12, interferon (IFN) $\gamma$, chemokine (C-X-C motif) ligand (CXCL) 9, CXCL10, CXCL11, prostaglandin (PG) E2, PGD2, and 15d-PGJ2 due to its impact on regulators upstream of inflammatory protein production [4-10]. Known mechanisms by which LMWF5A exerts its immunomodulatory effects include decreasing pro-inflammatory transcription factor activity (nuclear factor KB [NFKB] and signal transducer and activator of transcription [STAT]) [9], increasing antiinflammatory transcription factor activity (peroxisome proliferator-activated receptor [PPARY] and aryl 
hydrocarbon receptor [AhR]) [7, 9], and reducing endothelial cell permeability [11]. These modes of action predict that LMWF5A attenuates inflammation, promotes the resolution of immune responses, and restores homeostasis, supporting its use in the treatment of various inflammatory conditions.

In vitro data on the immunomodulatory activity of LMWF5A are substantiated by its in vivo clinical effects. The efficacy and safety of LMWF5A have been proven in clinical trials for the treatment of the inflammatory condition osteoarthritis $(O A)$ of the knee [12-16]. OA patients, particularly those with severe disease, receiving a single intraarticular injection of LMWF5A experience a statistically significant reduction in pain and improvement in function, with fewer adverse events compared to patients receiving a control injection of saline $[13,15]$. In addition, LMWF5A is currently being assessed for the treatment of coronavirus disease 2019 (COVID-19) complications [17] (ClinicalTrials.gov/NCT04456452, NCT04606784, NCT04839965, NCT04868890, NCT04880161), in which the downstream effects of cytokine storm are observed both in the lung and systemically [18].

Similar to LMWF5A, corticosteroids function by reducing levels of inflammatory mediators and vascular permeability [19]. They have been long and widely used for their potent anti-inflammatory and immunosuppressive properties, and patients have benefitted; however, corticosteroid use can be accompanied by adverse events, depending on dosage and duration of use [20]. With respect to intraarticular corticosteroid use for OA, these agents have been reported to induce cartilage cytotoxicity and catabolism, ultimately resulting in disease progression [21]. In COVID-19 patients, oral or IV administration of the glucocorticoid dexamethasone (DEX) has been found to lower mortality rates when disease severity required supplemental oxygen or ventilation $[22,23]$ however, adverse events include secondary infections, reactivation of latent infections, avascular necrosis of the hip, hyperglycemia, and psychiatric symptoms [24]. Thus, while corticosteroids are effective in treating inflammatory conditions, their negative side effects highlight the need for safer alternatives.

In this report, an unbiased search for LMWF5A targets and comparable upstream regulators led to the determination that LMWF5A acts on similar, but not identical, targets to DEX. Differences in target number, directional regulation, and identity between LMWF5A and DEX may provide hints regarding the comparatively low incidence of adverse events in patients treated with LMWF5A.

As an immune cell model, peripheral blood mononuclear cells (PBMC) were stimulated under three different stimulation conditions to evaluate the effect of LMWF5A on cells polarized toward proinflammatory or anti-inflammatory macrophage and lymphocyte phenotypes [25]. Differentially expressed genes between LMWF5A and vehicle control were determined by RNA sequencing, and results were subjected to an unbiased in silico analysis to 1) discover overall biological trends observed upon LMWF5A treatment and 2) compare or contrast known upstream regulator activity, i.e., compounds that act in a manner similar or opposite to LMWF5A. Further investigation into the most correlated upstream regulator, DEX, was accomplished by evaluating the common downstream targets of this compound and LMWF5A, and through comparison with public datasets of gene expression analyses in DEX-treated PBMC. 


\section{Methods}

\section{Cell treatment and RNA isolation}

Frozen human PBMC (ZenBio, Durham, NC, USA) were thawed and resuspended in XVivo 15 medium (Lonza, Verviers, Belgium). $50 \mu \mathrm{L}$ of $2 \times 10^{6}$ cells $/ \mathrm{mL}\left(1 \times 10^{5}\right.$ cells/well final) were treated with $50 \mu \mathrm{L}$ vehicle control (saline; KD Medical, Columbia, MD, USA) or LMWF5A (Ampio Pharmaceuticals, Englewood, CO, USA) in 96-well plates. Cells were then incubated at $37^{\circ} \mathrm{C}$ and $5 \% \mathrm{CO}_{2}$ for $1 \mathrm{~h}$. Immunostimulation was achieved by the addition of $10 \mu \mathrm{L}$ lipopolysaccharide (LPS; final concentration of $100 \mathrm{ng} / \mathrm{mL}$; Sigma, St. Louis, MO, USA), $10 \mu \mathrm{L}$ LPS + IFNY (final concentration of $100 \mathrm{ng} / \mathrm{mL}$ LPS and $20 \mathrm{ng} / \mathrm{mL}$ IFNy; R\&D Systems, Minneapolis, MN, USA), or $10 \mu \mathrm{L}$ IL-4 + IL-13 (final concentration of 10 $\mathrm{ng} / \mathrm{mL}$ each; R\&D Systems). Cells were further incubated at $37^{\circ} \mathrm{C}$ and $5 \% \mathrm{CO}_{2}$ for $24 \mathrm{~h}$. Cells were pelleted by centrifugation at $1000 \mathrm{rpm}$ for $10 \mathrm{~min}$. Medium was removed, and cells were processed for RNA isolation using the miRNeasy Mini Kit (Qiagen, Hilden, Germany). Ten technical treatment replicates per plate were combined after Qiazol addition to ensure that a sufficient amount of RNA was isolated for downstream analysis. This protocol was repeated three times on different days using a single, representative PBMC donor.

\section{RNA sequencing and differential expression analysis}

RNA samples were subjected to RNAseq by Lexogen (Vienna, Austria). Libraries were prepared using the QuantSeq 3' mRNA-Seq FWD Library Prep Kit (Lexogen). Sequencing was performed on a NextSeq 500 System (Illumina, San Diego, CA, USA) using a High Output Kit (v2, 75 cycles; Illumina) in single read mode.

Differential gene expression (DEG) between saline and LMWF5A treatments was achieved by comparing the following groups: LMWF5A + LPS vs. saline + LPS, LMWF5A + LPS/IFNy vs. saline + LPS/IFNy, LMWF5A + IL-4/IL-13 vs. saline + IL-4/IL-13. DESeq2 [26] analysis of raw RNA sequencing counts was performed in $\mathrm{R}$ [27] to determine transcript $\log _{2}$ fold-changes, $p$-values, and adjusted p-values. Genes with an adjusted $p$-value $<0.05$ were considered DEGs.

\section{In silico pathway analysis of LMWF5A DEGs}

Calculated DEG values were uploaded into Ingenuity Pathway Analysis (IPA) software (Qiagen Digital Insights, Redwood City, CA, USA). An Expression Core Analysis was run based on log ratios with an adjusted $p$-value $<0.05$ using the IPA Knowledge Base as reference. Graphical summaries were generated with the default node settings for each individual analysis [28]. Upstream regulators were computationally inferred and predicted to have similar or opposite actions to LMWF5A on the PBMC transcriptome [29]. Overlap p-values were derived from the Benjamini-Hochberg adjusted p-values; $\log$ (adjusted $p$-value) $>1.3$ was used as a significance cutoff. Z-scores where $z>0$ predicts activation or similar regulation, and $z<0$ predicts the converse; an absolute value of 2 was used as a significance cutoff. 


\section{In silico comparison of LMWF5A DEGs with public datasets}

Two publicly available DEX-treated PBMC datasets were used for comparison analysis. "Genetic mapping with multiple levels of phenotypic information reveals new determinants of lymphocyte glucocorticoid sensitivity" is a microarray dataset [30] generated from PBMC from each of 85 individual volunteers treated, in parallel, with $2.5 \mu \mathrm{g} / \mathrm{mL}$ phytohemagglutinin (PHA) and either vehicle (EtOH) or $1 \mu \mathrm{M}$ DEX for 6 h. DEG analysis comparing PHA + EtOH versus PHA + DEX was performed in Geo2R using default settings [27].

The second dataset entitled "Transcriptome analysis of porcine PBMCs reveals lipopolysaccharideinduced immunomodulatory responses and crosstalk of immune and glucocorticoid receptor signaling" [31] was generated from RNA sequencing of porcine PBMC treated with $10 \mu \mathrm{g} / \mathrm{mL}$ LPS and either vehicle (EtOH) or $5 \mathrm{nM}$ DEX for $2 \mathrm{~h}$. LPS + EtOH versus LPS + DEX conditions were used as a comparison to our datasets. The dataset was generated directly from supplemental data that had been analyzed by DESeq2 in $\mathrm{R}$ by the authors [27].

As IPA recommends that $<1000$ DEGs are used as input for analyses, a $\log _{2}$ fold change cutoff of -0.4 down and 0.4 up, in addition to an adjusted $p<0.05$, was employed. This resulted in 966 and 573 DEGs from the human and porcine PBMC datasets, respectively, to be used for subsequent comparison analysis. The LMWF5A PBMC datasets were reanalyzed with the same parameters $\left(\log _{2}\right.$ fold change cutoff of -0.4 down and 0.4 up, adjusted $p<0.05$ ), and an upstream regulator comparison analysis was performed in IPA.

\section{Results}

\section{Effect of LMWF5A on gene expression in stimulated PBMC}

RNA sequencing datasets from this study provide a snapshot of all genes that are regulated by LMWF5A in PBMC compared to saline vehicle control under three immunostimulatory conditions after 24 hours of treatment. The number of DEGs (adjusted $p<0.05$ ) were 55, 64, and 139 for the three following comparisons, respectively: LMWF5A + LPS vs. saline + LPS, LMWF5A + LPS/IFNy vs. saline + LPS/IFNy, and LMWF5A + IL-4/IL-13 vs. saline + IL-4/IL-13. A list of DEGs for each of the three conditions, their base means, $\log _{2}$ fold changes, $p$-values, and adjusted p-values are provided in Supplemental Tables 1-3.

\section{Overall biological trends upon LMWF5A treatment}

RNA sequencing results in an extensive, complicated dataset that can be difficult to interpret holistically, highlighting the importance of in silico analysis tools. To understand overall biological trends, IPA software employs the extensive IPA Knowledge Base, a database curated from empirical evidence in published manuscripts on the relationships, downstream effects, and connections of proteins, chemicals, and other molecules, to form a master network of biologically relevant connections. A 'core' IPA analysis algorithmically interrogates DEGs within a dataset of interest and predicts canonical pathways, upstream 
regulators, and biological functions that explain directional regulation of the observed DEGs within that dataset [29]. Graphical summaries highlight the most significant findings in the IPA core analysis and, in this study, predict the main biological targets and downstream activities of LMWF5A in an immune cell model. The graphical summary from PBMC stimulated with LPS/IFNY is shown as a representative network (Fig. 1); summaries for the LPS and IL-4/IL-13 conditions are shown in Supplemental Fig. 1a and b.

Several nodes are common between the three stimulation conditions. All nodes corresponding to biological targets represent molecules that are important in immune responses, such as cytokines, pattern recognition receptors, and inflammation-related transcription factors. Each graphical summary predicts inhibition of multiple interferons (IFNy, IFNa2), interleukins (IL-1 $\beta$, IL-6), TNF, the hormone and cytokine prolactin (PRL), toll-like receptors (TLRs; TLR7), and transcription factors (interferon regulatory factor [IRF]7, STAT1). In addition, many implicated biological functions are associated with a decrease in pro-inflammatory activities. These include the general immune response of cells, cytotoxicity, monocyte/macrophage phagocytosis, integrin-mediated signaling, as well as the development, trafficking, and adhesion of various immune cell types.

Furthermore, one pathway demonstrates predicted activation with LMWF5A treatment in IL-4/IL-13stimulated PBMC, the macrophage-stimulating protein (MSP)- recepteur d'origine nantais (RON) signaling pathway, which has been described as an important negative regulator of inflammation due to its inhibition of pro-inflammatory cytokine production by macrophages [32].

\section{Correlation of upstream regulators with LMWF5A}

Identification of molecules that act in a similar or opposite manner to LMWF5A is valuable to further understand how LMWF5A acts on immune cells. The IPA upstream regulator analysis uses the IPA Knowledge Base to interrogate user dataset DEGs and their directionality of regulation and identifies molecules that are predicted to produce either similar or dissimilar regulation of targets. Two measurements of confidence in the prediction are the overlap p-value and z-score. The overlap p-value is based on the proportion of the user dataset DEGs that overlaps with known targets of the upstream regulator. The $z$-score considers whether the directionality of the user dataset DEGs are consistent with known directional regulation by the upstream regulator (Fig. 2a). A comparison analysis of predicted upstream regulators was performed to visualize regulators that are relevant to all stimulation conditions. Specifically, IPA was used to calculate z-scores for upstream regulators in each condition, and the zscores for each upstream regulator were then summed for each condition and ranked by total z-score. Fig. $2 b$ lists the top upstream regulators predicted to match directionality with LMWF5A-regulated transcripts, while Fig. $2 \mathrm{c}$ lists the top regulators that are predicted to recapitulate gene regulation in the opposite direction of LMWF5A.

Interestingly, the upstream regulator with the highest total z-score was the glucocorticoid DEX (Fig. 2b), which acts as a glucocorticoid receptor agonist and affects a multitude of indirect downstream targets [33]. The primary action of DEX is to suppress inflammatory cell activity and inflammatory mediator 
levels, which mirrors the biological trends predicted by IPA for LMWF5A described above as well as previously published work on LMWF5A. The other top five most positively correlated upstream regulators with LMWF5A (Fig. 2b) include sirtuin 1 (SIRT1), prostaglandin E receptor 4 (PTGER4), filgrastim, and SB203580. Like DEX, each of these compounds has demonstrated anti-inflammatory activity.

In contrast, the top five upstream regulators that are predicted to result in gene expression changes that do not match with LMWF5A activity and have the lowest total z-scores are lipopolysaccharide (LPS), IFNy, STAT1, poly rl:rC-RNA, and IFNa2 (Fig. 2c). This list consists of established pro-inflammatory pathogen- and damage-associated molecular patterns (P/DAMP), transcription factors, and cytokines.

\section{Comparison of DEX and LMWF5A targets}

In the three different immunostimulatory conditions used to evaluate the effect of LMWF5A on PBMC, DEX was the most significant positively correlated upstream regulator to LWMF5A. Due to this finding and the longstanding and widespread utility of DEX in the clinic, a detailed comparison of its targets to the LMWF5A datasets was performed.

IPA upstream analysis determined that LMWF5A regulates 26,29 , and 42 established DEX targets for the LPS, LPS/IFNy, and IL-4/IL-13 stimulation conditions, respectively. Common targets represent a large proportion of each LMWF5A dataset, with $41 \%, 53 \%$, and $30 \%$ of transcripts significantly regulated by LMWF5A are also targeted by DEX (overlap -log(p-values): LPS, 10.2; LPS/IFNy, 9.4; IL-4/IL-13, 8.4). However, based on IPA computational modeling, directional regulation by LMWF5A of 10, 7, and 17 of common targets were inconsistent with that of DEX for the LPS, LPS/IFNy, and IL-4/IL-13 stimulation conditions. Nevertheless, overall, IPA modeling predicted significantly similar regulation of downstream molecules by DEX and LMWF5A based on directionally correlated transcripts (z-scores: LPS, 3.1; LPS/IFNy, 2.6; IL-4/IL-13, 2.4). Common targets between DEX and LMWF5A are shown for the LPS/IFNY stimulation conditions in a representative figure (Fig. 3). The common targets for the LPS and IL-4/IL-13 conditions are presented in Supplemental Fig. 2a and b.

In Fig. 3, 29 transcripts are presented that were regulated by LMWF5A in LPS/IFNy-stimulated PBMC at $24 \mathrm{~h}$ that are also known targets of DEX. Of these transcripts, 26 were decreased by LMWF5A (green) and 3 were increased (red). Further, of the 29 transcripts, 19 were directionally consistent with the action of DEX (orange or blue lines) and 7 were directionally inconsistent (yellow lines); 3 DEX targets have unpredicted directional regulation (gray lines).

Further comparison of DEX and LMWF5A revealed 71 total common targets (Fig. 4), and of these, only five transcripts were found to be affected by LMWF5A under all stimulation conditions: $\beta$-actin (ACTB), $\gamma^{-}$ actin (ACTG1), myristoylated alanine-rich C-kinase substrate (MARCKS), and tryptophanyl-tRNA synthetase 1 (WARS1), all associated with the cytoskeleton, and cytochrome b- $245 \beta$ chain (CYBB; also called NADPH oxidase 2), which functions to produce reactive oxygen species to eliminate pathogens during infection and is linked to the development of acute respiratory distress syndrome [34, 35]. Ten targets were shared between the datasets containing LPS, and three were shared between the IL-4/IL-13 
and each of the LPS and LPS/IFNy datasets. Moreover, 50 of the 71 common targets were unique to one stimulation condition, highlighting the wide-ranging effects of LMWF5A as well as its different activities depending on the inflammatory milieu. This result suggests that LMWF5A treatment may be beneficial in multiple inflammatory environments.

\section{Comparison of LMWF5A and DEX datasets}

The identification of DEX in our unbiased search for upstream regulators that correlate with LMWF5A prompted us to compare our datasets from stimulated PBMC treated with LMWF5A to published datasets of DEGs in stimulated PBMC treated with DEX. The GEO dataset entitled, "Genetic mapping with multiple levels of phenotypic information reveals new determinants of lymphocyte glucocorticoid sensitivity" [30] is a DEG analysis of PBMC treated with PHA $(2.5 \mu \mathrm{g} / \mathrm{mL})$ and either vehicle $(E t O H)$ or DEX $(1 \mu \mathrm{M})$ for $6 \mathrm{~h}$. Because PHA is a broad pro-inflammatory stimulant that acts as a strong lymphocyte mitogen as well as a ligand for multiple TLRs [36], it was deemed necessary to also compare the datasets from the current study to a dataset from PBMC that were stimulated in a similar manner (with LPS) and treated with DEX. Due to our inability to find a comprehensive gene expression analysis dataset of human PBMC stimulated with LPS and DEX, we performed a comparative analysis using a porcine PBMC model treated with $10 \mu \mathrm{g} / \mathrm{mL}$ LPS and either vehicle (EtOH) or $5 \mathrm{nM}$ DEX for $2 \mathrm{~h}$. As presented in Figure 5 and Supplemental Figure 3, respectively, we observed continuity in significant z-scores and adjusted overlap p-values for many upstream regulators when comparing LMWF5A- and DEX-treated PBMC datasets.

\section{Discussion}

In this study, an unbiased investigation was performed to predict biological targets and activities of LMWF5A in an immune cell model. This was accomplished by determining DEG upon LMWF5A treatment of stimulated PBMC followed by in silico analysis with IPA software. Evaluation of graphical summaries generated by IPA revealed that the overall biological trend observed in PBMC stimulated with LPS, LPS/IFNy, or IL-4/IL-13 in the presence of LMWF5A compared to saline control is the suppression of immune cell inflammatory activities, as numerous key pro-inflammatory regulators and functions were predicted to be inhibited and some anti-inflammatory regulators were predicted to be activated with LMWF5A. These predictions support the potential use of LMWF5A in a range of clinical conditions that result from a hyperactive or chronic immune response.

To delineate the predicted effects of LMWF5A on immune cells more specifically, known molecules with similar or opposite activities to LMWF5A were identified via upstream regulator analysis in IPA. The most similar upstream regulators were DEX, SIRT1, PTGER4, filgrastim, and SB203580. The glucocorticoid DEX, as a synthetic steroid, has multiple indications, including inflammatory conditions (such as rheumatoid and psoriatic arthritis, systemic lupus erythematosus, and Crohn's disease), multiple sclerosis, cerebral edema, shock, and allergies, amongst others [37]. Importantly, DEX has also been administered as an intra-articular injection for joint inflammation [37] and has been recently studied as a potent anti- 
inflammatory for the treatment of COVID-19 [22]; both conditions are currently being investigated in LMWF5A clinical trials.

SIRT1 is an NAD-dependent protein deacetylase that negatively regulates inflammation by altering cytokine levels and immune cell recruitment and activation by deacetylating and suppressing transcription factors, including NFKB [38]. Similarly, LMWF5A has also been proven to modulate transcription factor activity to decrease pro-inflammatory cytokine levels. Its ability to reduce TNF and IL$1 \beta$ release has been linked to its effects on the NFKB-repressing PPARY and AhR pathways in LPSstimulated PBMC, and it has also been shown to prevent NFKB reporter activity [9].

PTGER4 (or EP4) is a transmembrane, G-coupled protein receptor that becomes activated when bound to the cyclooxygenase (COX) pathway product PGE2, which is induced during inflammation. Although the role of PGE2 is pleotropic, it exerts its anti-inflammatory effects via PTGER4. PTGER4 binds to EP4 receptor-associated protein, which in turn, reduces the phosphorylation and increases the stability of p105, an inhibitor of NFKB and mitogen-activated protein/extracellular signal-regulated kinase (MEK) [39]. Overall, PTGER4 downregulates inflammation by modulating macrophage cytokine and chemokine secretion as well as T cell proliferation, differentiation, and cytokine production [40]. LMWF5A has been associated with COX pathway upregulation and its products, including the PTGER4 ligand PGE2, in PBMC and primary human osteoarthritic cells $[5,10]$. The mode of action of LMWF5A may be unique to the antiinflammatory drug class, as most inhibit both cytokine and prostaglandin release while LMWF5A inhibits cytokine release but promotes prostaglandin release. Inhibition of the COX/prostaglandin pathway can result in harmful side effects [41], and stimulation of this pathway with concomitant inhibition of cytokine production by LMWF5A may, conversely, promote anti-inflammation, resolution, and healing.

Filgrastim, recombinant human granulocyte colony stimulating factor, is commonly used clinically as a complement to chemotherapy due to its ability to stimulate granulocyte production, thus preventing low white blood cell counts [42]. In addition, this protein has confirmed anti-inflammatory properties in vivo with respect to the cytokine response and has been suggested as a potential treatment for chronic inflammatory conditions; for example, LPS-induced cytokine release has been shown to be attenuated in healthy human volunteers treated with filgrastim $[43,44]$.

Finally, SB203580 is a potent p38 mitogen-activated protein kinase (MAPK) inhibitor, with strong effects on cytokine production [45]. The p38 MAPK signaling pathway is critical to the regulation of many cellular processes, including inflammation, as it is activated in response to inflammatory mediators and other stress-related molecules and acts as a major regulator of cytokine production [46]. Overall, the top upstream regulators that significantly match LMWF5A activity have been empirically proven to downregulate components critical to inflammation, including pro-inflammatory cytokines and transcription factors.

The identification of upstream regulators with opposing activity to LMWF5A revealed LPS, poly rl:rC-RNA, IFNy, IFNa2, and STAT1 as the molecules with the most disparate effects. All of these molecules are known promoters of inflammation, as recognition of PAMPs and DAMPs by pattern recognition receptors, 
like TLRs, results in signal transduction to turn on multiple transcription factors (NF-KB, MAPK, STAT) that increase expression of key pro-inflammatory cytokines [1].

LPS and poly rl:rC-RNA are both categorized as PAMPs and function as signals of infection to initiate immune signaling via TLRs. LPS is a major component of Gram-negative bacterial cell walls, and acts as a PAMP upon bacterial infection. It is widely utilized in vitro to stimulate the TLR4-induced immune response that can occur upon recognition of either pathogens or endogenous molecules released upon tissue damage [47]. LPS was used in two of the three conditions in this study to stimulate an immune response in $\mathrm{PBMC}$, and its ranking as the most negatively correlated upstream regulator to LMWF5A highlights the fact that LMWF5A strongly counteracts TLR4-mediated inflammation. Moreover, the antiinflammatory effects of LMWF5A on cytokine release and transcription factor activity have been extensively studied using cells treated with LPS as a TLR4 stimulant $[7,9,48]$. poly rl:rC-RNA is a dsRNA mimic that can simulate infection with a dsRNA virus and activate TLR3 [49], suggesting that LMWF5A may also offset the actions of other TLR-driven pathways; the relationships between LMWF5A and other TLRs are currently under investigation.

As additional opposing upstream regulators, the cytokines IFNY and IFNa2 represent both classes of interferons, type II and I, respectively. They are secreted upon viral infection to limit viral replication and regulate the subsequent immune response [50]. The Janus kinase (JAK)-STAT signaling pathway is the most studied IFN-related transcription factor pathway, but IFNs also activate other signaling cascades, including p38 MAPK and phosphatidylinositol 3'-kinase, to exert their anti-viral and pro-inflammatory effects [51].

Because both type I and II IFNs activate STAT complexes, it is not surprising that STAT1 is also part of this list of opposing upstream regulators. Upon recognition of IFNs, a variety of interleukins, or other cytokines, STAT1 is phosphorylated, mainly by JAK kinases, and activated to drive a pro-inflammatory cascade [52]. LMWF5A was also previously demonstrated to inhibit the ability of STAT1 to bind its cognate DNA sequence in LPS-stimulated PBMC, implicating regulation of this transcription factor as part of the LMWF5A mode of action [9]. Inhibition of the JAK-STAT pathway is an effective therapeutic strategy for the treatment of inflammatory conditions, including rheumatoid arthritis (RA), psoriasis, inflammatory bowel disease [53] as well as COVID-19 [54]. In summary, the analysis of inversely correlated upstream regulators of LMWF5A represent pro-inflammatory factors, emphasizing the antiinflammatory activity of LMWF5A.

Comparison of the common targets between DEX and LMWF5A highlighted that a large proportion, onethird to one-half depending on the stimulation conditions, of the genes regulated by LMWF5A are also regulated by DEX. While most of the genes were regulated in a similar direction to DEX, others were directionally inconsistent.

An example of a target that is regulated by LMWF5A in a direction consistent with DEX is C-C motif chemokine ligand 2 (CCL2), also called monocyte chemoattractant-1. Upon recognition of inflammatory stimuli, CCL2 expression is induced, and this chemokine drives migration of immune cells, particularly 
monocytes, to the site of infection or tissue injury [55]. Dysregulated, increased CCL2 expression is linked to the pathology of many diseases, including heart failure, RA, and diabetes, due its overpromotion of immune cell infiltration and downstream pro-inflammatory effects [56]. Serum CCL2 has been demonstrated to be increased in $O A$ patients versus healthy controls, suggesting its importance to $O A$ pathogenesis [57]. Some studies have reported increased CCL2 levels in OA synovial fluid as well, and CCL2 has been linked to OA-associated pain in addition to factors influencing cartilage catabolism [58]. With respect to COVID-19, CCL2, along with many other inflammatory cytokines, contributes to the cytokine storm that occurs during the body's dysregulated response to SARS-CoV2 infection and its level has been correlated with increased disease severity [54]. Thus, the similar activities of both DEX and LMWF5A on CCL2 should provide benefit to patients with CCL2-related diseases, including OA and COVID-19.

Conversely, an example of a target that is regulated by LMWF5A in a direction inconsistent with DEX is cathepsin B (CTSB). CTSB is member of the cathepsin family of cysteine proteases, which are localized in the lysosome [59]. It is well studied in the context of cancer [60] but has also been implicated in cartilage degradation and $\mathrm{OA}$ pathogenesis due to its proteolytic activity of extracellular matrix components and its ability to promote the activity of other proteases [61]. In fact, the enzymatic activity of CTSB was demonstrated to be enhanced in vitro in stimulated primary chondrocytes and in vivo in cartilage, serum, and synovial fluid from OA patients, in which CTSB activity levels were associated with OA disease severity [62]. In the IPA upstream regulator analysis, the IPA Knowledge Database records CTSB as being upregulated by DEX, supporting the cartilage-degrading effects observed with corticosteroids, while the differential expression analysis showed that CTSB was downregulated by LMWF5A. This and other differences may be important distinctions between these two anti-inflammatory treatments. Data comparing LMWF5A directly to DEX in PBMC have been published for a small number of targets. These data showed that in LPS-stimulated PBMC, DEX inhibited TNF, PGE2, and 15d-PGJ2, whereas LMWF5A inhibited TNF but increased levels of PGE2 and 15d-PGJ2 by ELISA [10]. While it is possible to explore each specific directionally consistent or inconsistent target, the present analysis allows for a broad understanding of overall LMWF5A activity and its comparison to DEX as well as the interplay between common targets and their downstream pathways.

The differences in common targets between these datasets points to a limitation of the current study. This analysis solely examined the effects of LMWF5A in one cell model at one time point, albeit with three immunostimulation conditions. Additional timepoints or cell types would provide even further insight into the mode of action of LMWF5A and may identify more overlapping targets. For example, due to the known effects of LMWF5A on cytokine release from PBMC, an earlier timepoint may have captured more differentially expressed cytokine RNAs as well as any temporal differences in cytokine RNA levels due to LMWF5A treatment.

Although all molecules identified in these analyses shed light on the putative effects of LMWF5A, the distinction that DEX was scored by IPA as the most similar upstream regulator prompted further analysis of the LMWF5A datasets with public datasets of DEGs in stimulated PBMC treated with DEX. Of the top 
32 upstream regulators as identified in the comparison analysis between DEX and LMWF5A datasets and ranked by absolute z-score, only 11 had $>2$ LMWF5A datasets with a non-significant z-score compared to the DEX datasets (Fig. 5). In addition, the observed z-scores and adjusted p-values were not as strongly significant for each of the LMWF5A datasets compared to the DEX datasets, supporting the hypothesis that while LMWF5A acts similarly to DEX, it is not identical to DEX.

Extensive information on the MOA of LMWF5A has been gained with the datasets in this investigation, allowing for the formation of multiple hypotheses that can be tested in future experiments. For instance, targeted experiments exploring the effects of LMWF5A will be performed using DEX as a comparative treatment. These experiments will focus on components of various signaling pathways, including IFNmediated signaling due to the high instance of IFN-related factors that were predicted to be affected by LMWF5A, including IRFs, STAT1, and both type I and II IFNs themselves. In addition, several specific, inflammatory disease-associated, directionally consistent, and inconsistent targets of LMWF5A and DEX will be investigated, for example CCL2 and CTSB as mentioned above.

\section{Conclusion}

Unbiased in silico analysis of RNA sequencing datasets from activated PBMC treated with the biologic drug LMWF5A compared to vehicle control demonstrates that LMWF5A may target numerous inflammation-related factors and functions, ultimately resulting in a putative suppression of proinflammatory and a potential promotion of pro-resolving immune cell activities. Upstream regulator analysis identified DEX as the most positively correlated molecule to LMWF5A. Due to significant overlap between their targets, LMWF5A may be indicated for the many conditions in which DEX is used. This glucocorticoid and LMWF5A exhibit extensive anti-inflammatory effects due to their ability to simultaneously inhibit multiple cytokines by affecting upstream activity. This differs from other treatments of acute and chronic inflammatory conditions that inhibit individual cytokines, such as antibody-based medicines. In addition, DEX targets many molecules that are not regulated by LMWF5A, and some of the targets that are regulated by both are inversely affected. These observations may help to explain why DEX is associated with side-effects that are not experienced by LMWF5A-treated patients. Furthermore, this study supports our previous research and provides new hypotheses for future investigations on the mode of action of LMWF5A.

\section{Declarations}

\section{Funding}

Funding for this work was provided by Ampio Pharmaceuticals.

\section{Competing Interests}

E.D.F. is an employee and has been granted stock options at Ampio Pharmaceuticals. M.A.H. is a consultant, shareholder, and has been granted stock options at Ampio Pharmaceuticals. G.W.T. is an 
employee, shareholder, and has been granted stock options at Ampio Pharmaceuticals. D.B-O. is a member of the board of directors and shareholder at Ampio Pharmaceuticals.

\section{Author contributions}

E.D.F.: Conception; experimental design and execution; data acquisition, analysis, and interpretation; drafting of manuscript. M.A.H.: Conception; data acquisition, analysis, and interpretation; drafting of manuscript. G.W.T.: Critical review and revision of the manuscript. D.B-O.: Conception; project supervision; data interpretation; critical review and revision of the manuscript. All authors have read and approved the manuscript.

\section{Ethics approval and consent to participate}

All experiments were conducted with commercially available primary human cells, with all appropriate consents available from the supplier.

Consent for publication

Not applicable.

Availability of data and material

Full datasets will be uploaded to Gene Expression Omnibus upon manuscript acceptance.

Code availability

Code is available from the corresponding author upon request.

\section{Acknowledgements}

The authors acknowledge technical support from Lexogen $\mathrm{GmbH}$ for library preparation and sequencing.

\section{References}

1. Chen L, Deng H, Cui H, Fang J, Zuo Z, Deng J, Li Y, Wang X, Zhao L (2018) Inflammatory responses and inflammation-associated diseases in organs. Oncotarget 9:7204-7218

2. Medzhitov R, Horng T (2009) Transcriptional control of the inflammatory response. Nat Rev Immunol 9:692-703

3. Bar-Or D, Thomas G, Rael LT, Frederick E, Hausburg M, Bar-Or R, Brody E (2019) On the Mechanisms of Action of the Low Molecular Weight Fraction of Commercial Human Serum Albumin in Osteoarthritis. Curr Rheumatol Rev 15:189-200

4. Bar-Or D, Thomas GW, Bar-Or R, Rael LT, Scarborough K, Rao N, Shimonkevitz R (2006) Commercial human albumin preparations for clinical use are immunosuppressive in vitro. Crit Care Med 34:1707-1712 
5. Frederick ED, Hausburg MA, Thomas GW, Rael LT, Brody E, Bar-Or D (2016) The low molecular weight fraction of human serum albumin upregulates $\mathrm{COX} 2$, prostaglandin $\mathrm{E} 2$, and prostaglandin $\mathrm{D} 2$ under inflammatory conditions in osteoarthritic knee synovial fibroblasts. Biochem Biophys Rep 8:68-74

6. Hausburg MA, Frederick ED, McNair P, Schwappach J, Banton KL, Roshon M, Lieser MJ, Acuna DL, Banerjee RR, Bar-Or D (2018) Clinically relevant redifferentiation of fibroblast-like chondrocytes into functional chondrocytes by the low molecular weight fraction of human serum albumin.Clin Exp Rheumatol36:891-895

7. Rael LT, Bar-Or R, Banton KL, Mains CW, Roshon M, Tanner AH, Lieser MJ, Acuna DL, Bar-Or D (2018) The anti-inflammatory effect of LMWF5A and N-acetyl kynurenine on macrophages: Involvement of aryl hydrocarbon receptor in mechanism of action. Biochem Biophys Rep 15:61-67

8. Shimonkevitz R, Thomas G, Slone DS, Craun M, Mains C, Bar-Or D (2008) A diketopiperazine fragment of human serum albumin modulates T-lymphocyte cytokine production through rap1. $J$ Trauma 64:35-41

9. Thomas G, Frederick E, Thompson L, Bar-Or R, Mulugeta Y, Hausburg M, Roshon M, Mains C, Bar-Or D (2020) LMWF5A suppresses cytokine release by modulating select inflammatory transcription factor activity in stimulated PBMC. J Transl Med 18:452

10. Thomas GW, Rael LT, Hausburg M, Frederick ED, Mains CW, Slone D, Carrick MM, Bar-Or D (2016) The low molecular weight fraction of human serum albumin upregulates production of $15 \mathrm{~d}-\mathrm{PGJ} 2$ in Peripheral Blood Mononuclear Cells. Biochem Biophys Res Commun 473:1328-1333

11. Thomas GW, Rael LT, Hausburg M, Frederick ED, Brody E, Bar-Or D (2016) The low molecular weight fraction of commercial human serum albumin induces acetylation of alpha-tubulin and reduces transcytosis in retinal endothelial cells. Biochem Biophys Res Commun 478:1780-1785

12. Bar-Or D, Salottolo KM, Loose H, Phillips MJ, McGrath B, Wei N, Borders JL, Ervin JE, Kivitz A, Hermann M, Shlotzhauer T, Churchill M, Slappey D, Clift V (2014) A randomized clinical trial to evaluate two doses of an intra-articular injection of LMWF-5A in adults with pain due to osteoarthritis of the knee. PLoS ONE 9:e87910

13. Cole B, McGrath B, Salottolo K, Bar-Or D (2018) LMWF-5A for the Treatment of Severe Osteoarthritis of the Knee: Integrated Analysis of Safety and Efficacy. Orthopedics 41:e77-e83

14. McGrath B (2015) Unique aspects of pain reduction in osteoarthritis of the knee with LMWF-5A. Open Access Rheumatol 7:19-22

15. Salottolo K, Cole B, Bar-Or D (2018) Intra-articular injection of the anti-inflammatory compound LMWF-5A in adults with severe osteoarthritis: a double-blind prospective randomized controlled multi-center safety and efficacy trial. Patient Saf Surg 12:11

16. Schwappach J, Dryden SM, Salottolo KM (2017) Preliminary Trial of Intra-articular LMWF-5A for Osteoarthritis of the Knee. Orthopedics 40:e49-e53

17. Thomas G, Frederick E, Hausburg M, Goldberg L, Hoke M, Roshon M, Mains C, Bar-Or D (2020) The novel immunomodulatory biologic LMWF5A for pharmacological attenuation of the "cytokine storm" in COVID-19 patients: a hypothesis. Patient Saf Surg 14:21 
18. Tang L, Yin Z, Hu Y, Mei H (2020) Controlling Cytokine Storm Is Vital in COVID-19. Front Immunol 11:570993

19. Barnes PJ (2006) How corticosteroids control inflammation: Quintiles Prize Lecture 2005. Br J Pharmacol 148:245-254

20. Yasir M, Goyal A, Bansal P, Sonthalia S (2021) Corticosteroid Adverse Effects. In StatPearls

21. Wernecke C, Braun HJ, Dragoo JL (2015) The Effect of Intra-articular Corticosteroids on Articular Cartilage: A Systematic Review. Orthop J Sports Med 3:2325967115581163

22. Ahmed MH, Hassan A (2020) Dexamethasone for the Treatment of Coronavirus Disease (COVID-19): a Review.SN Compr Clin Med1-10. https://doi.org/10.1007/s42399-020-00610-8

23. Group RC, Horby P, Lim WS, Emberson JR, Mafham M, Bell JL, Linsell L, Staplin N, Brightling C, Ustianowski A, Elmahi E, Prudon B, Green C, Felton T, Chadwick D, Rege K, Fegan C, Chappell LC, Faust SN, Jaki T, Jeffery K, Montgomery A, Rowan K, Juszczak E, Baillie JK, Haynes R, Landray MJ (2021) Dexamethasone in Hospitalized Patients with Covid-19. N Engl J Med 384:693-704

24. Chen F, Hao L, Zhu S, Yang X, Shi W, Zheng K, Wang T, Chen H (2021) Potential Adverse Effects of Dexamethasone Therapy on COVID-19 Patients: Review and Recommendations. Infect Dis Ther 1-25. https://doi.org/10.1007/s40121-021-00500-z

25. Martinez FO, Sica A, Mantovani A, Locati M (2008) Macrophage activation and polarization. Front Biosci 13:453-461

26. Love MI, Huber W, Anders S (2014) Moderated estimation of fold change and dispersion for RNA-seq data with DESeq2. Genome Biol 15:550

27. R-Core-Team (2021) R: A language and environment for statistical computing. R Foundation for Statistical Computing, Vienna, Austria

28. Qiagen-Ingenuity (2021) Graphical Summary Algorithm

29. Kramer A, Green J, Pollard J Jr, Tugendreich S (2014) Causal analysis approaches in Ingenuity Pathway Analysis. Bioinformatics 30:523-530

30. Maranville JC, Baxter SS, Witonsky DB, Chase MA, Di Rienzo A (2013) Genetic mapping with multiple levels of phenotypic information reveals determinants of lymphocyte glucocorticoid sensitivity. Am J Hum Genet 93:735-743

31. Li Z, Trakooljul N, Hadlich F, Ponsuksili S, Wimmers K, Murani E (2021) Transcriptome analysis of porcine PBMCs reveals lipopolysaccharide-induced immunomodulatory responses and crosstalk of immune and glucocorticoid receptor signaling. Virulence 12:1808-1824

32. Huang L, Fang X, Shi D, Yao S, Wu W, Fang Q, Yao H (2020) MSP-RON Pathway: Potential Regulator of Inflammation and Innate Immunity. Front Immunol 11:569082

33. Newton R (2000) Molecular mechanisms of glucocorticoid action: what is important? Thorax 55:603-613

34. Carnesecchi S, Pache JC, Barazzone-Argiroffo C (2012) NOX enzymes: potential target for the treatment of acute lung injury. Cell Mol Life Sci 69:2373-2385 
35. Diebold BA, Smith SM, Li Y, Lambeth JD (2015) NOX2 As a Target for Drug Development: Indications, Possible Complications, and Progress. Antioxid Redox Signal 23:375-405

36. Unitt J, Hornigold D (2011) Plant lectins are novel Toll-like receptor agonists. Biochem Pharmacol $81: 1324-1328$

37. Lexicomp (ed) Dexamethasone. UpToDate

38. Vachharajani VT, Liu T, Wang X, Hoth JJ, Yoza BK, McCall CE (2016) Sirtuins Link Inflammation and Metabolism. J Immunol Res 2016:8167273

39. Minami M, Shimizu K, Okamoto Y, Folco E, llasaca ML, Feinberg MW, Aikawa M, Libby P (2008) Prostaglandin E receptor type 4-associated protein interacts directly with NF-kappaB1 and attenuates macrophage activation. J Biol Chem 283:9692-9703

40. Tang EH, Libby P, Vanhoutte PM, Xu A (2012) Anti-inflammation therapy by activation of prostaglandin EP4 receptor in cardiovascular and other inflammatory diseases. J Cardiovasc Pharmacol 59:116-123

41. Ong CK, Lirk P, Tan CH, Seymour RA (2007) An evidence-based update on nonsteroidal antiinflammatory drugs. Clin Med Res 5:19-34

42. Frampton JE, Lee CR, Faulds D (1994) Filgrastim. A review of its pharmacological properties and therapeutic efficacy in neutropenia. Drugs 48:731-760

43. Hartung T (1999) Anti-inflammatory aspects of Filgrastim and impact on IL-2 release. J Hematother Stem Cell Res 8(Suppl 1):S21-S22

44. Hartung T, Doecke WD, Bundschuh D, Foote MA, Gantner F, Hermann C, Lenz A, Milwee S, Rich B, Simon B, Volk HD, von Aulock S, Wendel A (1999) Effect of filgrastim treatment on inflammatory cytokines and lymphocyte functions. Clin Pharmacol Ther 66:415-424

45. Cuenda A, Rouse J, Doza YN, Meier R, Cohen P, Gallagher TF, Young PR, Lee JC (1995) SB 203580 is a specific inhibitor of a MAP kinase homologue which is stimulated by cellular stresses and interleukin-1. FEBS Lett 364:229-233

46. Yang Y, Kim SC, Yu T, Yi YS, Rhee MH, Sung GH, Yoo BC, Cho JY (2014) Functional roles of p38 mitogen-activated protein kinase in macrophage-mediated inflammatory responses. Mediators Inflamm 2014:352371

47. Lu YC, Yeh WC, Ohashi PS (2008) LPS/TLR4 signal transduction pathway. Cytokine 42:145-151

48. Thomas GW, Rael LT, Mains CW, Slone D, Carrick MM, Bar-Or R, Bar-Or D (2016) Anti-Inflammatory Activity in the Low Molecular Weight Fraction of Commercial Human Serum Albumin (LMWF5A). J Immunoassay Immunochem 37:55-67

49. Alexopoulou L, Holt AC, Medzhitov R, Flavell RA (2001) Recognition of double-stranded RNA and activation of NF-kappaB by Toll-like receptor 3. Nature 413:732-738

50. Lee AJ, Ashkar AA (2018) The Dual Nature of Type I and Type II Interferons. Front Immunol 9:2061. https://doi.org/10.3389/fimmu.2018.02061 
51. Platanias LC (2005) Mechanisms of type-l- and type-II-interferon-mediated signalling. Nat Rev Immunol 5:375-386

52. Ramana CV, Chatterjee-Kishore M, Nguyen H, Stark GR (2000) Complex roles of Stat1 in regulating gene expression. Oncogene 19:2619-2627

53. Banerjee S, Biehl A, Gadina M, Hasni S, Schwartz DM (2017) JAK-STAT Signaling as a Target for Inflammatory and Autoimmune Diseases: Current and Future Prospects. Drugs 77:521-546

54. Chen Y, Wang J, Liu C, Su L, Zhang D, Fan J, Yang Y, Xiao M, Xie J, Xu Y, Li Y, Zhang S (2020) IP-10 and MCP-1 as biomarkers associated with disease severity of COVID-19. Mol Med 26:97

55. Deshmane SL, Kremlev S, Amini S, Sawaya BE (2009) Monocyte chemoattractant protein-1 (MCP-1): an overview. J Interferon Cytokine Res 29:313-326

56. Gschwandtner M, Derler R, Midwood KS (2019) More Than Just Attractive: How CCL2 Influences Myeloid Cell Behavior Beyond Chemotaxis. Front Immunol 10:2759

57. Ni F, Zhang Y, Peng X, Li J (2020) Correlation between osteoarthritis and monocyte chemotactic protein-1 expression: a meta-analysis. J Orthop Surg Res 15:516

58. Scanzello CR (2017) Chemokines and inflammation in osteoarthritis: Insights from patients and animal models. J Orthop Res 35:735-739

59. Turk V, Stoka V, Vasiljeva O, Renko M, Sun T, Turk B, Turk D (2012) Cysteine cathepsins: from structure, function and regulation to new frontiers. Biochim Biophys Acta 1824:68-88

60. Aggarwal N, Sloane BF (2014) Cathepsin B: multiple roles in cancer. Proteomics Clin Appl 8:427-437

61. Baici A, Lang A, Zwicky R, Muntener K (2005) Cathepsin B in osteoarthritis: uncontrolled proteolysis in the wrong place. Semin Arthritis Rheum 34:24-28

62. Ben-Aderet L, Merquiol E, Fahham D, Kumar A, Reich E, Ben-Nun Y, Kandel L, Haze A, Liebergall M, Kosinska MK, Steinmeyer J, Turk B, Blum G, Dvir-Ginzberg M (2015) Detecting cathepsin activity in human osteoarthritis via activity-based probes. Arthritis Res Ther 17:69

\section{Figures}



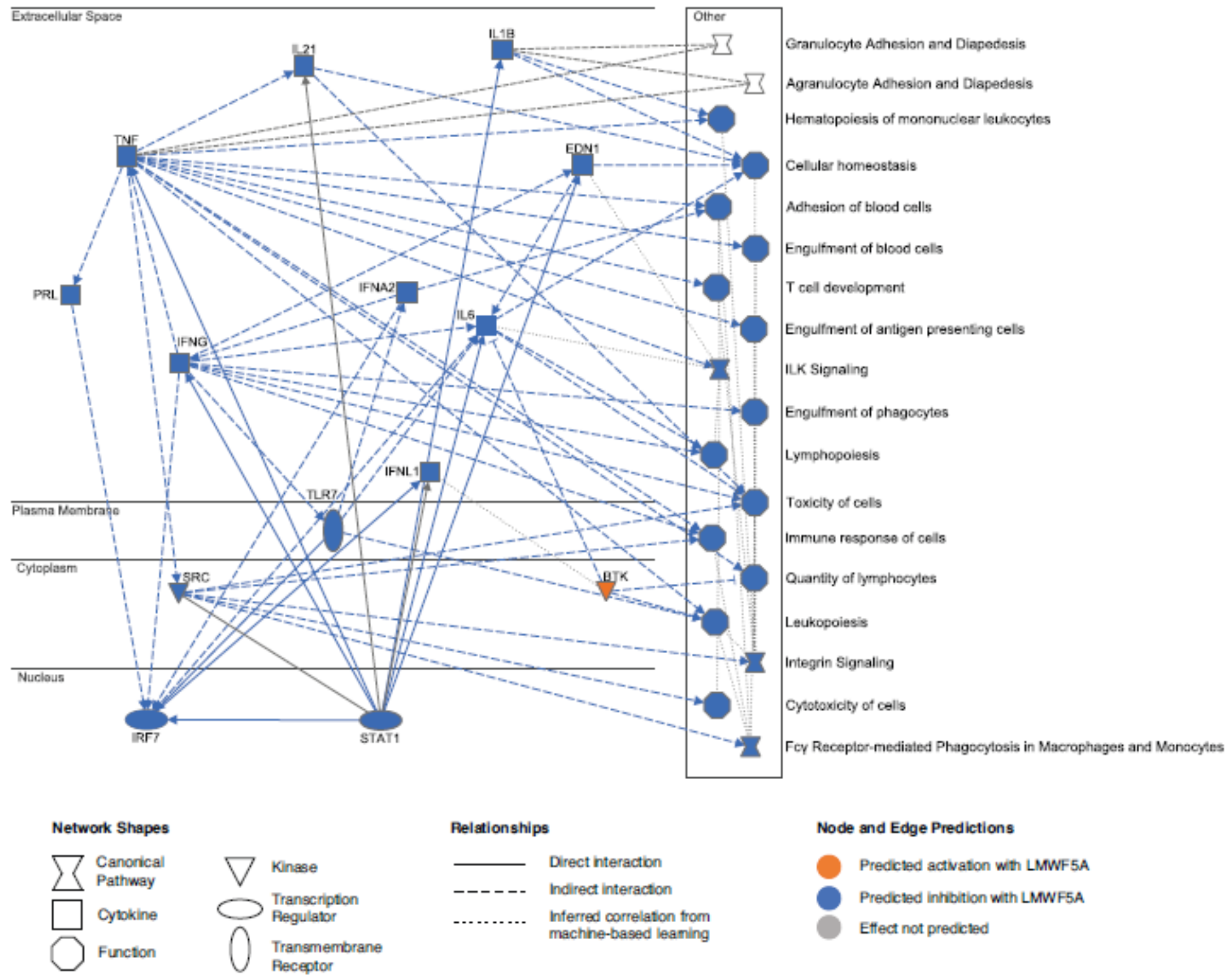

Figure 1

Graphical summary of effects of LMWF5A on LPS/IFNY-stimulated PBMC. Differential expression analysis comparing LMWF5A and saline treatment in LPS/IFNY-stimulated PBMC were uploaded into IPA software, and the graphical summary algorithm was run with the 'default node' and 'subcellular localization' settings to visualize the overall effects of LMWF5A treatment. Each node located in the cellular compartments represents a gene that is predicted to be significantly activated or inhibited by LMWF5A. The nodes located in the 'other' section represent canonical pathways or functions that are predicted to be significantly activated or inhibited. The shape of each node represents the functional class of the regulator, pathway, or function. The node color corresponds to its predicted activation (orange) or inhibition (blue); no color indicates that while there is an effect, its direction cannot be predicted by IPA. For edges, a solid line denotes a direct physical interaction between two nodes, while a dotted line denotes an indirect functional interaction, and arrows denote activation, while bars denote inhibition as referenced in the IPA Knowledge Database. 


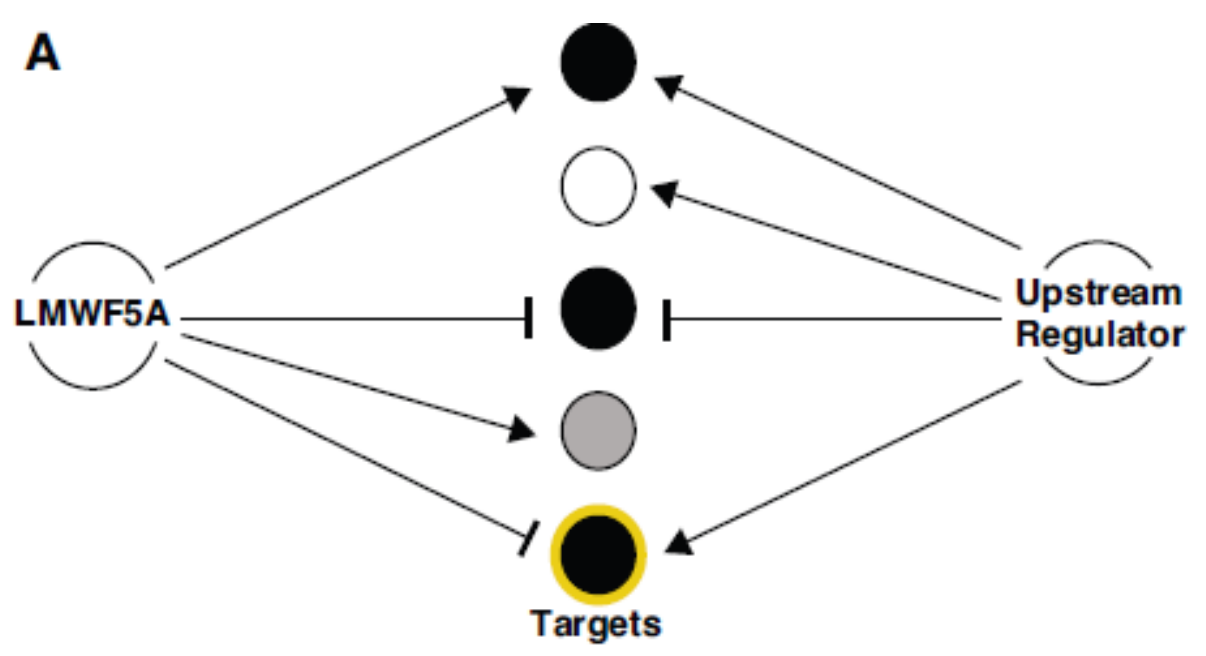

B

\begin{tabular}{|l|c|c|c|c|}
\multirow{2}{*}{ upstream regulator } & \multicolumn{3}{|c|}{ z-score (overlap p-value) } & \multirow{2}{*}{ total z-score } \\
\cline { 2 - 5 } & LPS & LPS/FNy & IL-4/L-13 & \\
\hline dexamethasone & $3.1(9.4)$ & $2.6(10.2)$ & $2.4(8.4)$ & 8.1 \\
\hline SIRT1 & $2.2(2.9)$ & $2.2(2.7)$ & $2.7(3.3)$ & 7.1 \\
\hline PTGER4 & $2.4(4.1)$ & $2.0(3.0)$ & $2.0(1.2)$ & 6.4 \\
\hline filgrastim & $1.5(7.2)$ & $1.3(4.0)$ & $3.2(6.7)$ & 6.0 \\
\hline SB203580 & $3.1(5.0)$ & $2.9(6.2)$ & N/A & 6.0 \\
\hline
\end{tabular}

\begin{tabular}{l}
\multicolumn{5}{c|}{ Z } \\
\begin{tabular}{|l|c|c|c|c|}
\multirow{2}{*}{ upstream regulator } & \multicolumn{3}{|c|}{ Z-score (overlap p-value) } & \multirow{2}{*}{ total z-score } \\
\cline { 2 - 4 } & LPS & LPSAFNy & IL-4/IL-13 & \\
\hline lipopolysaccharide & $-4.5(20.5)$ & $-4.4(23.6)$ & $-3.3(15.8)$ & -12.2 \\
\hline IFNG & $-4.0(14.3)$ & $-4.1(19.2)$ & $-3.2(15.8)$ & -11.3 \\
\hline STAT1 & $-4.1(16.8)$ & $-3.1(6.3)$ & $-2.9(3.9)$ & -10.1 \\
\hline poly rl:rC-RNA & $-3.8(12.2)$ & $-3.4(8.1)$ & $-2.6(2.3)$ & -9.7 \\
\hline IFNA2 & $-3.7(13.8)$ & $-3.3(9.1)$ & $-2.0(2.5)$ & -9.0 \\
\hline
\end{tabular}
\end{tabular}

\section{Figure 2}

Upstream regulator analysis. (a) Concept of upstream regulator analysis. Differential expression datasets for LMWF5A versus saline in PBMC stimulated with LPS, LPS/IFNy, or IL-4/IL-13 were individually subjected to upstream regulator analysis. IPA was utilized to compare the observed expression changes in the datasets that were due to LMWF5A with known directional changes of targets by upstream regulators found in the literature and comprising the IPA Knowledge database. Upstream regulator analysis produces an overlap p-value and a z-score as measures of confidence in the prediction. The overlap p-value reflects the proportion of the user dataset that are also known upstream regulator targets; in this example, 3 out of the 4 DEGs changed by LMWF5A are also upstream regulator targets (black dots). The z-score is derived by interrogating whether the directionality of the targets match (positive zscore) or anti-match (negative z-score) with the known regulation by the upstream regulator according to the IPA Knowledge Base. Z-scores are affected by consistency within the data; in this example, one of the overlapping targets is activated by the upstream regulator but is decreased by LMWF5A so it is deemed 
inconsistent (black dot with yellow outline) and brings the z-score closer to zero. (b-c) Results of upstream regulator analysis. The top five molecules that were identified to match (b) or anti-match (c) with the activity of LMWF5A in the IPA upstream regulator analysis are listed. Statistical significance was determined by z-score, with a z-score of $>2$ representing a significant positive correlation and a z-score of $<-2$ representing a significant negative correlation. The tables are sorted by rows with the highest (b) or lowest (c) total z-score for all three stimulation conditions.
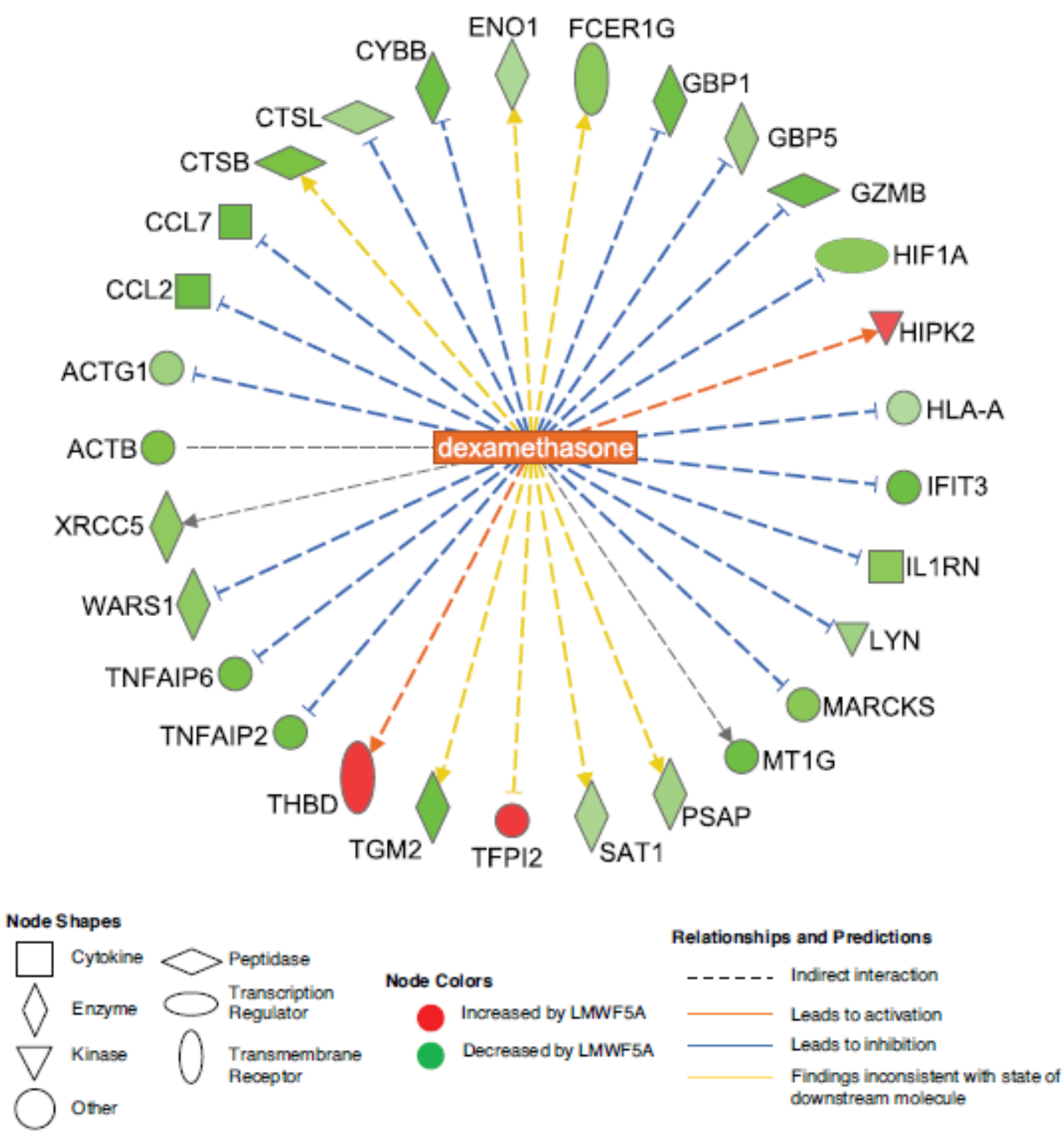

\section{Figure 3}

Common targets of DEX and LMWF5A in LPS/IFNY-stimulated PBMC. Upstream regulator analysis was performed in IPA on differentially expressed genes due to LMWF5A treatment versus saline in LPS/IFNYstimulated PBMC. DEX was selected and displayed as a network to visualize the 29 targets that are common to DEX and LMWF5A. Each node located on the outside of the wheel represents a gene that is targeted by both LMWF5A and DEX. The shape of the node indicates the functional class of the gene. The green nodes represent genes that are decreased by LMWF5A, while the red nodes represent genes 
that are increased by LMWF5A, and the intensity of the node color indicates the magnitude of this increase or decrease. For the edges connecting DEX with each gene target, arrows denote activation by DEX, while bars denote inhibition by DEX. The colors of the edges correspond to whether the effect of LWF5A on that target is directionally consistent or inconsistent with that of DEX (blue lines = directionally consistent and inhibited; orange lines = directionally consistent and activated; yellow lines = directionally inconsistent; gray lines = affected but direction unable to be predicted due to lack of data in the IPA Knowledge Base).

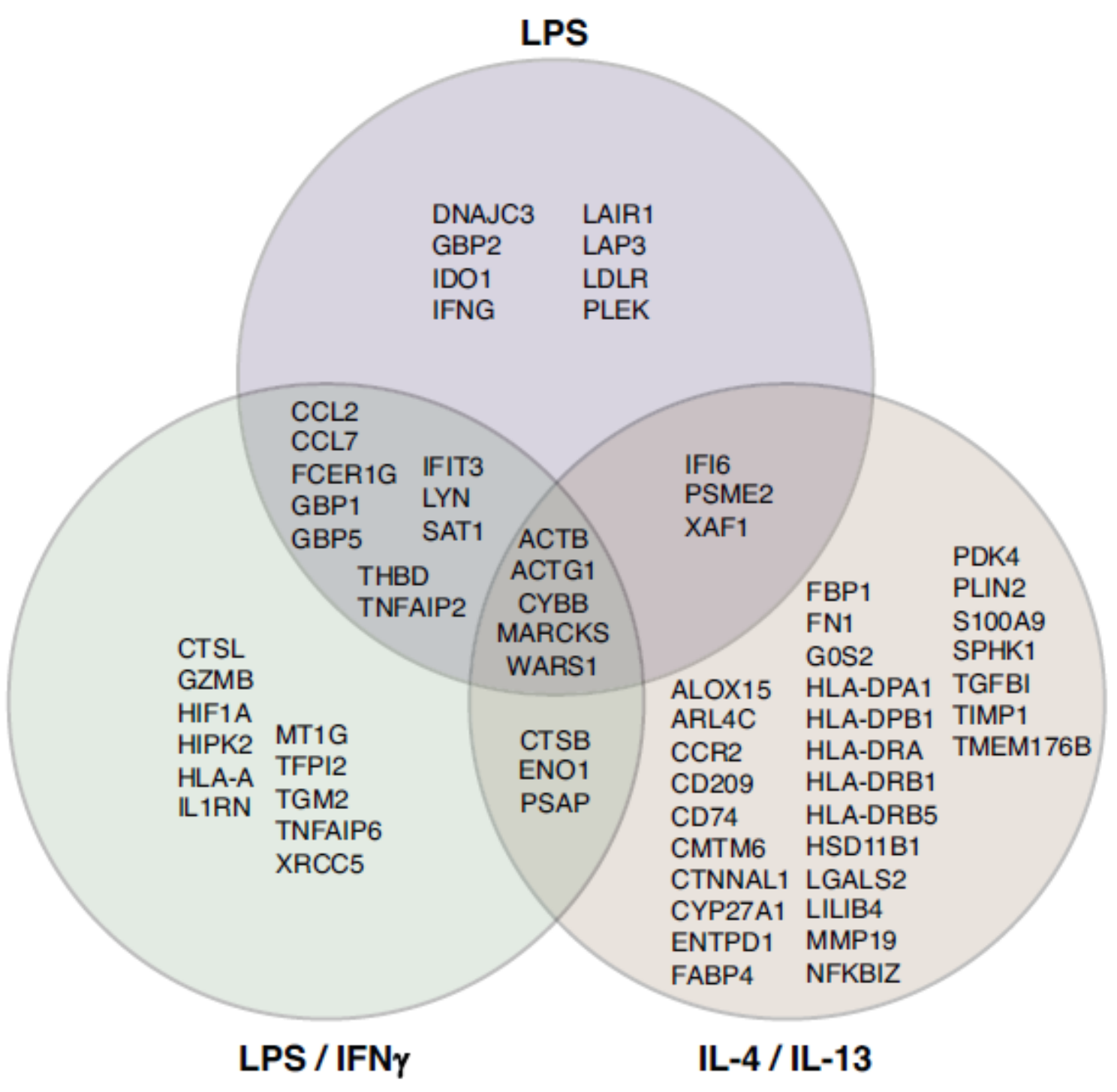

\section{Figure 4}

Comparison of PBMC stimulation conditions for LMWF5A/DEX targets. Targets of LMWF5A and DEX were identified by upstream regulator analysis in IPA as described in Figure 3. A Venn diagram was constructed to depict these targets and their overlap for PBMC stimulated with LPS, LPS/IFNy, or IL-4/IL13. 


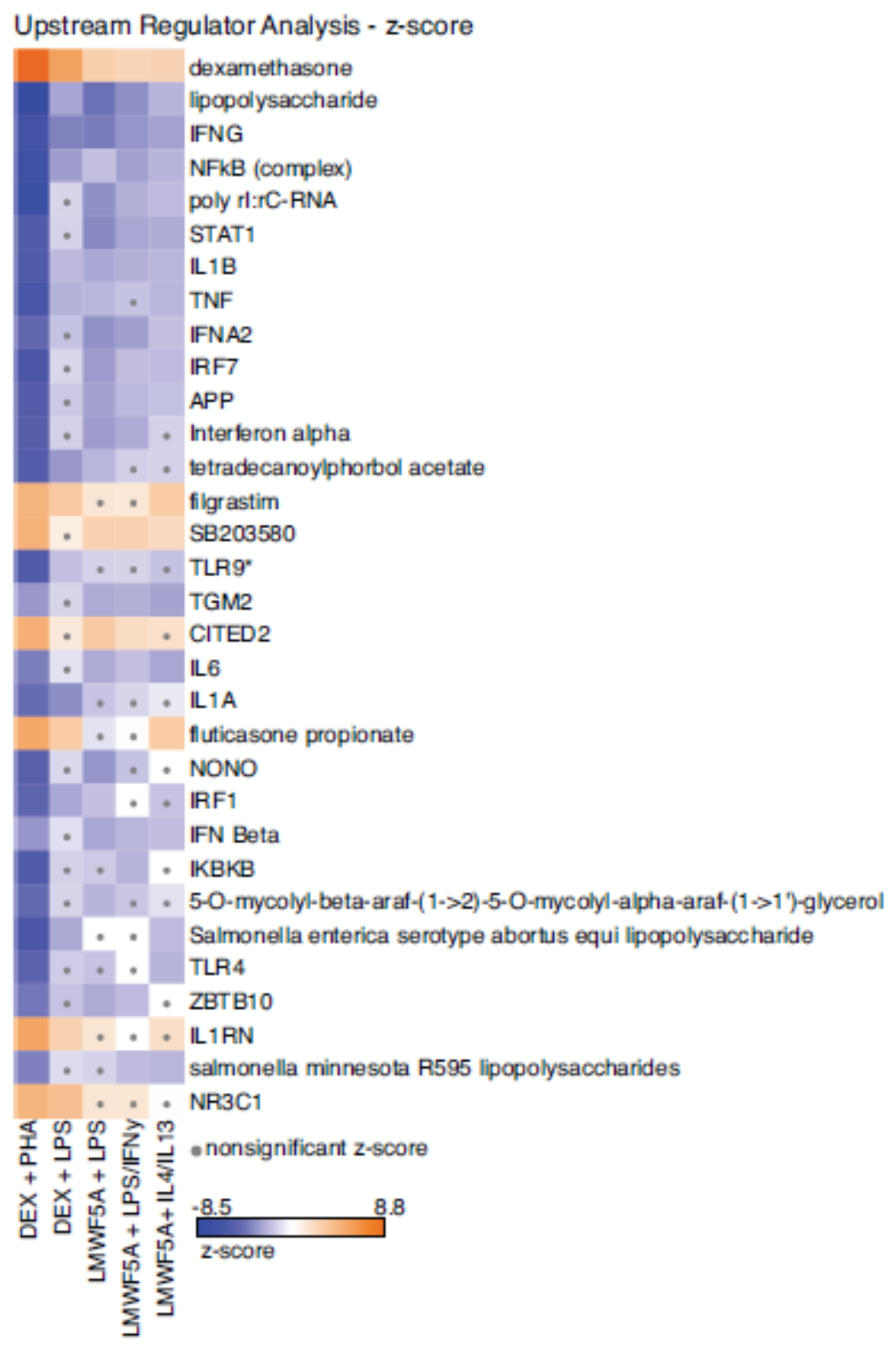

\section{Figure 5}

Comparison of datasets for LMWF5A treatment of PBMC with publicly available datasets for DEX treatment of PBMC. Upstream regulator analysis in IPA was utilized to compare the expression changes in the datasets that were due to LMWF5A (in human PBMC stimulated with LPS, LPS/IFNg, or IL-4/IL-13) or DEX (in human PBMC stimulated with PHA or in porcine PBMC stimulated with LPS) with known directional changes of targets by upstream regulators found in the literature and comprising the IPA Knowledge database. The identification of upstream regulator that matched (orange) or were antimatches (blue) to LMWF5A or DEX regulation of DEGs within the respective datasets. Statistical significance was determined by $z$-score, with a $z$-score of $>2$ representing a significant positive correlation and a $z$-score of $<-2$ representing a significant negative correlation. The upstream regulators are sorted by z-score (absolute value) of the DEX-treated, PHA-stimulated PBMC dataset.

\section{Supplementary Files}


This is a list of supplementary files associated with this preprint. Click to download.

- SupplementalTables13.xlsx

- SupplementalFigure1.eps

- SupplementalFigure2.eps

- SupplementalFigure3.eps

- SupplementalFigure4.eps

- SupplementalFigure5.eps 